\title{
Developing an Interactive 3D Learning Experience to Help Students Understand Key Regulatory Processes Associated with Glycolysis
}

Jacqueline Mason, Kevin Brennan, Samantha Bond, Leah Lebowicz

University of Illinois at Chicago, Chicago, IL

\section{Abstract}

Recent recommendations for undergraduate biology instruction emphasize teaching foundational biological principles and helping students transfer these principles to more complex biological phenomena. These curricular endeavors can be facilitated by the incorporation of interactive visualization materials. The following research project was developed to explore whether a visual interactive didactic tool could be developed to improve learning outcomes for undergraduate biology students on the topic of allosteric regulation within the context of glycolysis. The results of this research could be beneficia for improving the development of interactive applications for science education.

\section{Introduction \& Purpose}

Complex topics such as cellular respiration are often introduced before students have a solid understanding of foundational biological principles. This lack of understanding can hinder the development of critical thinking abilities needed for integrating increasingly complex scientific information as students continue their studies. Using glycolysis as the context, the purpose of this research project was to improve learning outcomes for introductory biology students studying cellular respiration by fostering foundational knowledge about enzymatic regulation. A 2D animation and a dynamic interactive application that allowed for real-time exploration of proteins and molecules were created. Content was presented in a manner that allowed students to build on prior information and transfer concepts to the specific context of glycolysis. Materials were made accessible to students as an online module.

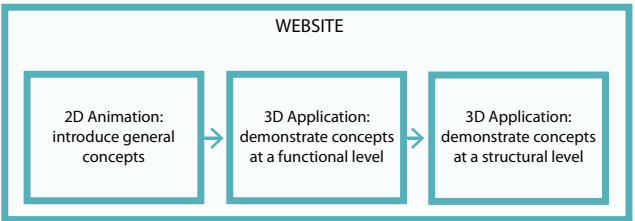

Figure 1: scaffolded knowledge building of biological principles

\section{Materials \& Methods}

Overall Process

Glycolytic enzymes with resolved structures were first aggregated from the Protein Data Bank and modified in the Visual Molecular Dynamics (NMD) software. Enzyme models were then exported to Pixologic ZBrush where they were retopologized for efficiency of
geometry and performance (see figure 3). Enzymes and substrates were animated in Autodesk $3 d$ s Max. The user interface elements were

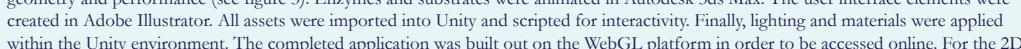
animation, all elements were created within Adobe lllustrator. Adobe After Efrces as used for compositing and the finished animation

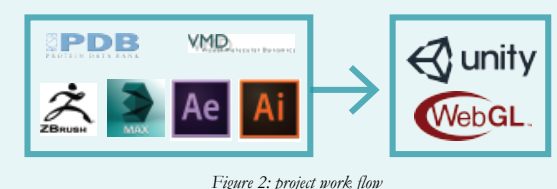

Figure 2: project work flow

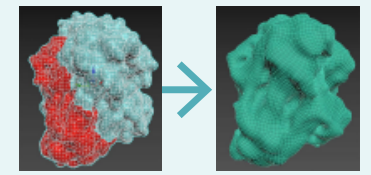

Figure 3: optimizing ensyme model geometry
Application Design and Delivery

There were two primary scenes in the application

Functional level scene (see figure 4): Students were able to control the amount of adenosine triphosphate (ATP) in the environment and observe the effects on enzymatic activity. By manipulating this value, allosteric enzymes were either activated or deactivated. User interface
elements were implemented strategically to help scaffold student understanding of this process. Students were then encouraged to click on the third enzyme, phosphoffuctokinase, to observe allosteric regulation at the structural leve.

Structural level scene (see figure 5): The final scene allowed students to interact with and observe the regulatory mechanisms of
phosphofructokinase (PFK). Students could again manipulate the levels of ATP in the system and observe the effects on PFK. At this level direct $\mathrm{s}$ (t) guidance to help them deconstruct and understand their experience with this part of the application. The finished application and $2 \mathrm{D}$ animation were optimized and exported for web delivery (see figures 6 and 7). The landing page contains a brief introduction along with the D animation. The next page hosts the interactive application

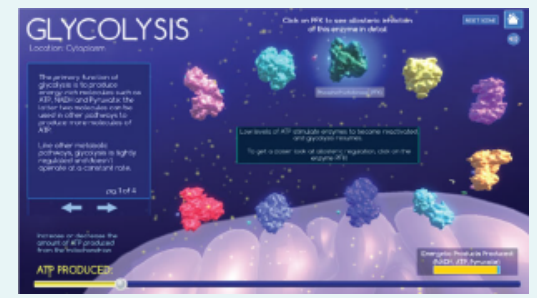

Figure 4: functional overview of feedlacke inhibition in ghrolysit

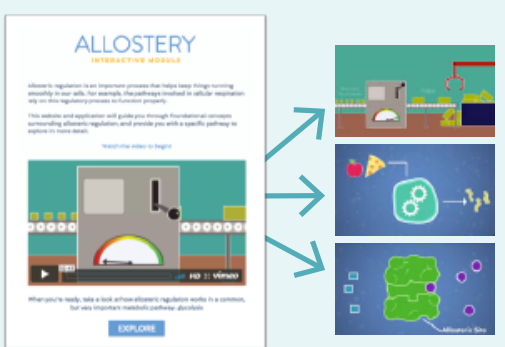

Figure 6: website landing page and selected animation screensbor

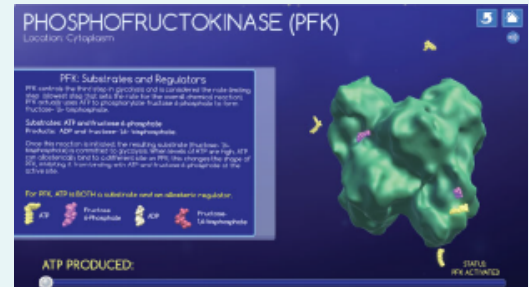

Figure 5: structural overview of allostertic requlation in $P F K$

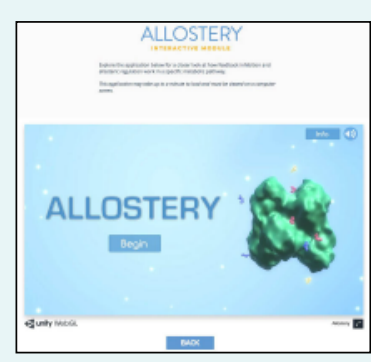

\section{Results}

A pretest-posttest design method was used during an introductory biology course at the University of Illinois at Chicago in Spring 2017. 16 students completed the pretest and 12 completed the posttest. Both tests were worth 5 points. A decrease of 1.38 points was observed for the average score on the posttest. Due to the lack of control with this research study and the smal sample sizes, it is not possible to draw meaningful conclusions from these preliminary quantitative results. Qualitative results aggregated from the posttest reveal an overall positive experience with the interactive application (see figure 8 below).

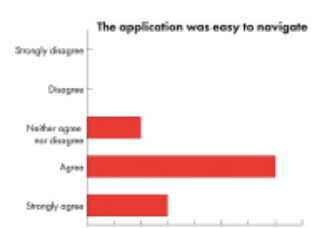

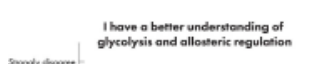
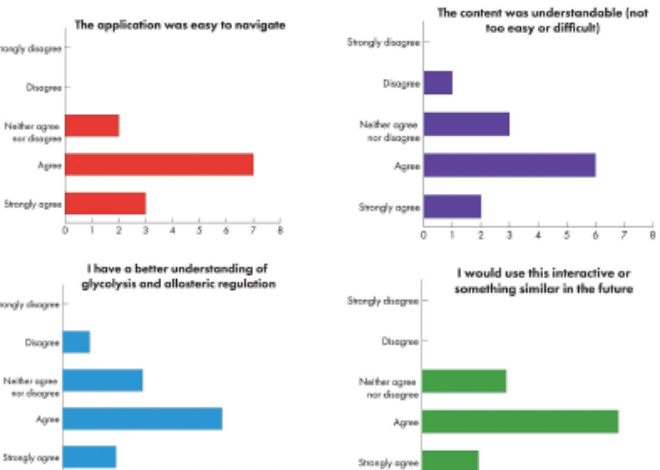

-

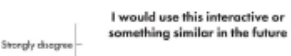

congere-

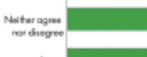

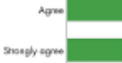

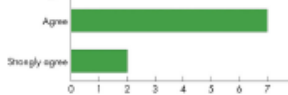

Figure 8: student qualitative feedback for the interactive application

\section{Discussion}

This project sought to combine effective teaching pedagogies with a novel interactive visual presentation in order to foster deeper understanding of allosteric regulation and glycolysis. When combined with interactivity, visualizations allow students to explore spatial and causal relationships in a dynamic and constructive manner, helping students build connections and integrate information. Future directions will focus on improving the overall design and conducting a more rigorous and accurate analysis of the application's efficacy.

\section{References}

Gilbert, J. K. 22007). Vsualization in Science Education. Dordrecht: Springer.

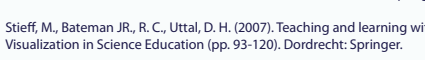

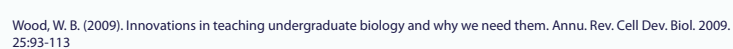

